\title{
CORRELAÇÃO ENTRE INTERNAÇÕES POR MICOSES E CONDIÇÕES CLIMÁTICAS EM SÃO LUÍS, NO PERÍODO DE 1998 A 2016
}

\section{CORRELATION BETWEEN HOSPITALIZATIONS FOR MYCOSES AND CLIMATIC CONDITIONS IN SÃO LUÍS, FROM 1998 TO 2016}

\author{
Letícia Chagas da Silva ${ }^{1}$; Jessflan Rafael Nascimento Santos ${ }^{2}$; Edilene de Araújo Diniz Pinheiro ${ }^{3}$; \\ Amanda Graziela Gonçalves Mendes ${ }^{4}$; Mayara Lucyanne Santos de Araújo ${ }^{5}$; \\ Juliana Lopes Almeida ${ }^{6}$; Fabrício Brito Silva ${ }^{7}$; Julliana Ribeiro Alves dos Santos ${ }^{8}$.
}

\begin{abstract}
RESUMO: As micoses sistêmicas representam um problema de saúde pública no Brasil e são doenças endêmicas em alguns estados, como no Maranhão. São causadas por fungos que estão presentes no ambiente associados ao solo, vegetais, materiais em decomposição e excretas de aves. As condições climáticas podem influenciar a proliferação e a disseminação destes agentes, contribuindo com o aumento do número de casos de internações. Neste contexto, o objetivo deste estudo foi avaliar a correlação entre as condições climáticas e as internações por micoses em São Luís (MA), no período de 1998 a 2016. Após a aprovação do Comitê de Ética em Pesquisa da Universidade CEUMA, a coleta de dados secundários referentes aos aspectos climáticos e de internações por micoses foi realizada em bases nacionais. A análise de Correlação de Spearman foi aplicada para os dados mensais de internações por micoses e precipitação ou temperatura máxima/ mínima. Foram registrados 786 casos de internações por micoses em São Luís, no período avaliado. Uma correlação positiva entre a precipitação $(p<0,05)$ e o número de internações por micoses foi observada, enquanto para as temperaturas mínima $(p<0,05)$ e máxima $(p>0,05)$, as correlações foram negativas. Os resultados deste estudo destacam que quanto maior a temperatura, menor o número de internações por micoses em São Luís. Este estudo destaca a importância da vigilância epidemiológica das micoses no Estado do Maranhão e da avaliação da modulação das condições ambientais na proliferação de fungos patogênicos.
\end{abstract}

PALAVRAS-CHAVE: São Luís, fungos, precipitação, temperatura.

\footnotetext{
${ }^{1}$ Mestre em Meio Ambiente, Universidade CEUMA. E-mail: let-chagas@ hotmail.com

${ }^{2}$ Mestre em Meio Ambiente, Universidade CEUMA. E-mail: jessflan@ymail.com

${ }^{3}$ Graduanda em Biomedicina, Universidade CEUMA. E-mail: edilene.diniz@live.com

${ }^{4}$ Graduanda em Biomedicina, Universidade CEUMA. E-mail: grazielamendes29@ gmail.com

${ }^{5}$ Engenheira Ambiental e Mestre em Geociências Aplicadas e Geodinâmica, Universidade de Brasília; Doutoranda em Engenharia Civil e Ambiental, Universidade Federal de Campina Grande. E-mail: mayara.araujo.eng@ gmail.com

${ }^{6}$ Engenharia Ambiental, Universidade CEUMA. E-mail: july01.jl@gmail.com

${ }^{7}$ Docente do Mestrado em Meio Ambiente da Universidade CEUMA. E-mail: fabricio.brito@ @euma.br

${ }^{8}$ Docente do Mestrado em Meio Ambiente da Universidade CEUMA. E-mail: julliana.santos@ @euma.br.
} 


\begin{abstract}
Systemic mycoses are a public health problem in Brazil and are endemic diseases in some states, such as Maranhão. The mycoses are caused by fungi that are present in the environment associated to soil, vegetables, decomposing materials and excreta of birds. Climatic conditions can influence the proliferation and dissemination of these agents, contributing to the increase in the number of hospitalization cases. In this context, the objective of this study was to evaluate the correlation between climatic conditions and hospitalizations for mycoses in São Luís (MA) from 1998 to 2016. After the approval of the Research Ethics Committee of the CEUMA University, the collection of secondary data regarding climatic aspects and hospitalizations for mycoses was carried out on a national basis. Spearman's correlation analysis was applied to the monthly data on admissions for mycoses and precipitation or maximum / minimum temperature. There were 786 cases of hospitalizations for mycoses in São Luís, during the period evaluated. A positive correlation between the precipitation $(p>0.05)$ and the number of admissions for mycoses was observed, while for the minimum $(p<0.05)$ and maximum $(p>0.05)$ temperatures, correlations were negative. The results showed that the higher the temperature, the lower the number of hospitalizations due to mycoses in São Luís. This study highlights the importance of epidemiological surveillance of mycoses in the State of Maranhão and the evaluation of the modulation of environmental conditions in the proliferation of pathogenic fungi.
\end{abstract}

KEY WORDS: São Luís, fungi, precipitation, temperature.

\title{
1 INTRODUÇÃO
}

Micoses Sistêmicas são infecções causadas por fungos patogênicos primários e que têm como porta de entrada o trato respiratório, a partir do qual podem disseminar para todo o organismo. As micoses sistêmicas endêmicas no Brasil são: Paracoccidioidomicose, Histoplasmose, Coccidioidomicose e Criptococose. A incidência de infecções fúngicas varia de acordo com as condições socioeconômicas, fatores geoclimáticos, hábitos culturais e o número de condições individuais de risco para a aquisição dessas infecções. A ausência de notificação compulsória dificulta a aquisição de dados epidemiológicos e mascara a incidência real de infecções fúngicas no país (Giacomazzi, 2015).

O município de São Luís está dentro da área compreendida como tropical zona equatorial, com elevados valores médios na temperatura, tendo a média acima de $18^{\circ} \mathrm{C}$ em todos os meses do ano, apresentando uma classificação climática pelo método de Thorntwaite tipo semi-úmido com 4 a 5 meses secos (EMBRAPA, 2013).

Para Mendonça \& Danni-Oliveira (2007, p. 159), o clima de São Luís recebe influência tanto da maritimidade quanto da continentalidade, apresentando pequena variação sazonal e a pluviosidade, maior que os índices térmicos, contendo duas estações bem definidas pelo regime pluviométrico com um período chuvoso entre o verão e outono, e outro seco, entre o inverno e a primavera. 
Além disso, a capital maranhense está situada em uma região de alta influência da Zona de Convergência intertropical (ZCIT) que é determinante para compreensão do regime pluviométrico de todo Nordeste brasileiro, tendo o período de maior influência a partir de janeiro com seu deslocamento meridional, estabelecendo um regime de pluviosidade mais regular nos meses de março e abril, com elevados índices de precipitação (FEITOSA, 1996).

Neste contexto, o objetivo deste estudo foi avaliar a correlação entre as internações por micoses e as condições climáticas, entre os anos de 1998 e 2016, em São Luís, Maranhão.

\section{MATERIAIS E MÉTODOS}

\section{1 Área de Estudo}

São Luís, capital do estado do Maranhão, possui uma área territorial de $834,785 \mathrm{~km}^{2}$, com uma população de 1.014.837 habitantes (IBGE, 2016). Compõe a ilha de São Luís, juntamente com os municípios de Paço do Lumiar, São José de Ribamar e Raposa. Possui uma posição geográfica privilegiada e estratégica, com o segundo mais profundo porto do mundo, o Porto de Itaqui. Detém um setor industrial desenvolvido, com destaque a empresa de mineração.

A capital do Maranhão localiza-se em uma área de transição entre a região Norte e Nordeste brasileira (Silva et al., 2016). Com uma grande diversidade de fauna e flora, e em destaque, está a vegetação litorânea da região. Alguns rios nascem na cidade, sendo o mais importante o Rio Bacanga. São Luís possui pequenas áreas de Floresta Amazônica, principalmente nas áreas dos parques ambientais.

\subsection{Aquisição dos dados}

O Projeto foi submetido e aprovado pelo Comitê de Ética em Pesquisa da Universidade CEUMA, sob o parecer número: 2.363.324. Os dados considerados neste estudo incluíram a base quantitativa de internações por micoses sistêmicas registradas no município de São Luís, no período de 1998 a 2016. A base municipal foi adquirida no site do Departamento de Informática do Sistema Único de Saúde (DATASUS) (DATASUS, 2017) e no Sistema de Informação Hospitalar (SIH) (SIH, 2017), ambos visam garantir uma maior organização e acessibilidade no processamento das informações estaduais e municipais relativas a internações hospitalares.

Os dados climatológicos foram adquiridos no site do Instituto Nacional de Meteorologia (INMET), por meio da estação meteorológica localizada na capital maranhense. Foram utilizados os 
registros anuais das variáveis de precipitação, temperatura máxima e mínima, em uma série temporal de 1998 a 2016.

Posteriormente, os dados de internações por micoses e os dados climáticos foram organizados em uma mesma planilha. Assim, foi possível aplicar a correlação de Spearman. O coeficiente de correlação linear de Spearman corresponde ao coeficiente de correlação de Pearson, depois de transformados pela equação de Wilcoxon (Spearman, 1904). O coeficiente de Spearman é usado, principalmente, para quantificar o quão duas colunas de dados dependem umas das outras. É baseado em uma classificação não paramétrica, que independe do tamanho e da distribuição dos dados (Zhang et al., 2016).

\section{RESULTADOS E DISCUSSÃO}

De acordo com a Tabela 1, a correlação de Spearman entre a precipitação e o número de internações por micoses indica uma correlação positiva $(\mathrm{p}<0,05)$, enquanto para as temperaturas mínima e máxima, a correlação de Spearman entre estas variáveis com o número de internações foi negativa, entretanto somente a temperatura mínima apresentou um valor $\mathrm{p}<0,05$. Dessa forma, quanto maior a temperatura, menor o número de internações (Tabela 1).

Tabela 1. Correlação de Spearman de hospitalizações por micoses com precipitação (ppt), temperatura mínima e temperatura máxima

\begin{tabular}{cccc}
\hline & ppt & Tmin & Tmax \\
\hline Spearman R & 0,1468 & $-0,1603$ & $-0,1336$ \\
Valor de P & 0,0267 & 0,0156 & 0,0532 \\
\hline
\end{tabular}

Fonte: Autores.

Em São Luís, o ano que obteve maior registros de internações por micoses foi 2004 com 153 casos. Neste ano, assim como no ano anterior a precipitação se manteve crescente antigindo 2.496,8 mm (Figura 1 A). Enquanto que no ano de 2005 foi um ano com a precipitação anual bem abaixo do normal com apenas $150,3 \mathrm{~mm}$, houve também um decréscimo nos registros de internações, mas ainda se mantiveram altos com 103 casos. Isso pode ter acontecido em função do ano anterior ter altos índices pluviométricos, período que pode ter ocorrido o contato com o agente fúngico, uma vez que o período de incubação das micoses sistêmicas pode variar de dias a anos.

Observa-se o mesmo comportamento nos anos seguintes, em 2010 com 87 casos, onde em 2009 temos o ano mais chuvoso da década com registro de 2.847,8 mm. Já a partir de 2012, nota-se uma considerável queda nos níveis de chuvas, da mesma forma, houve uma redução nos casos 
notificados de internação, o que pode estar associado ao aumento da temperatura máxima, pois no ano de 2011 foi o segundo ano mais chuvoso da década com registro de 2.713,3 $\mathrm{mm}$.

Figura 1. Correlação entre Internações por Micoses (I.M.) X Precipitação (A) e Correlação entre Internações por Micoses (I.M.) X Temperatura em São Luís, 1998 a 2016 (B)

A

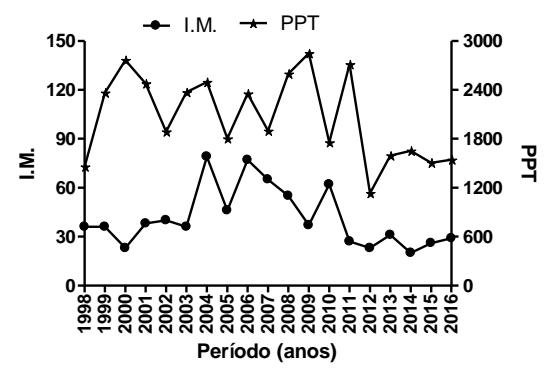

B

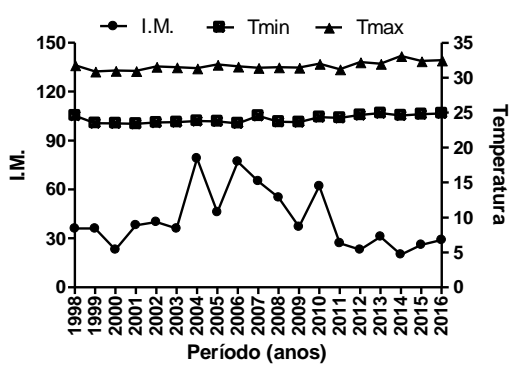

Fonte: Autores.

A média da temperatura máxima ao longo da série temporal é de $31,63^{\circ} \mathrm{C}$ e a média da temperatura mínima é de $24,17^{\circ} \mathrm{C}$, enquanto a média do número de internações ficou em torno de 71 casos. Observa-se que nos anos que houve maiores registros de notificações de internações, as temperaturas estavam abaixo da média (Figura 1B).

Em 2004, a média anual da temperatura máxima ficou em torno de $31,37^{\circ} \mathrm{C}$ e a média anual da temperatura mínima em $23,82^{\circ} \mathrm{C}$. Da mesma forma em 2006, exceto pela temperatura máxima, onde o registro ficou bem próximo da média com $31,61^{\circ} \mathrm{C}$.

Considerando os últimos 6 anos, observa-se que a média das temperaturas máxima e mínima aumentaram, ficando em torno de 32,19 e $27,70^{\circ} \mathrm{C}$, enquanto que o número de internações houve um decréscimo, ficando com o registro médio de 50 casos.

As condições ambientais têm um impacto importante na incidência de algumas infecções fúngicas. Uma epidemia de coccidioidomicose no Arizona foi associada às condições climáticas, enquanto a Califórnia experimentou uma epidemia na década de 1990 que possivelmente estava ligada a condições de estiagem (Park et al., 2005).

As ligações iniciais entre as condições climáticas e as infecções fúngicas foram identificadas há várias décadas. Recentemente, foram publicados mais detalhes sobre clima e a coccidioidomicose. Esses estudos identificaram associações ligando o clima e outros fatores aos padrões sazonais de coccidioidomicose e à variabilidade interanual e tendências da doença. As 
variáveis significativas incluíram índices de seca, precipitação retardada, temperatura, velocidade do vento e poeira (Kolivras e Comrie,2003).

Parte da razão para o pouco conhecimento tem sido a falta de séries de dados de doenças com uma alta qualidade. Um grande desafio para entender mais sobre os vínculos entre clima e doenças infecciosas continua a ser a dificuldade em obter séries temporais regulares de dados sobre infecções fúngicas. Na ausência de dados adequados sobre a variabilidade ambiental do próprio fungo, é necessário explorar dados epidemiológicos de diferentes maneiras para identificar melhor o papel dos fatores de controle ambiental, como o clima. Até o momento, os dados de incidência de doenças oferecem a melhor (e única) série temporal multianual disponível para comparação com as condições climáticas (CNP-EUA, 2001).

Sheppard et al. 2002 afirmam que uma melhor compreensão dos fatores climáticos por trás dos focos das infecções fúngicas permitirá um melhor tempo de amostragem ambiental para estas e medidas de mitigação relacionados a fatores ambientais e outros fatores que afetem os surtos e o potencial para o desenvolvimento de um sistema de aviso prévio antes de um surto. A capacidade de definir um evento crítico, como a precipitação, pode permitir procedimentos de mitigação imediatamente após o evento, além de fornecer uma útil ferramenta de saúde pública com um prazo de 18 meses na incidência esperada destas infecções.

\section{CONCLUSÕES}

Os dados apresentados confirmam que surtos de internações por micoses podem ser influenciados pelo clima, uma vez que os agentes etiológicos das micoses sistêmicas estão presentes no ambiente.

Os resultados mostraram que quanto maior a temperatura, menor o número de internações por micoses em São Luís. Este estudo destaca a importância da vigilância epidemiológica das micoses no Estado do Maranhão e da avaliação da modulação das condições ambientais na proliferação de fungos patogênicos. 


\section{REFERÊNCIAS}

Comitê Nacional de Pesquisas (EUA) sobre Clima, Ecossistemas, Doenças Infecciosas e Saúde Humana. 2001; Dignani, M.C. Epidemiologia de doenças fúngicas invasivas com base nos relatórios de autópsias. F1000Prime Rep 2014; 6:81.

DATASUS. Departamento de Informática do Sistema Único de Saúde. 2017. Acesso a Informação. Disponível em: <http://datasus.saude.gov.br/datasus/quem-e-quem> Acesso em: 01 nov. 2017.

EMPRESA BRASILEIRA DE PESQUISA AGRIOECYPARUA - EMBRAPA. Relatório do diagnóstico do macrozoneamento ecológico-econômico do Estado do Maranhão.

BATISTELLA, M.; BOLFE, E.L.; VICENTE, L.E.; VICTORIA, D. DE C., ARAUJO, L.S. (Org.). - Campinas, SP: Embrapa, 2013. 325 p.

IBGE. Instituto Brasileiro de Geografia e Estatística. Cidades. Disponível em:

<http://cod.ibge.gov.br/2VT3Y> Acesso em: 01 dez. 2017.

KOLIVRAS, K.N.; COMRIE, A.C. Modelando a incidência da febre do vale com base em condições climáticas no condado de Pima, no Arizona. Int J Biometeorol. 2003; 47: 87-101.

MENDONÇA, Francisco e DANNI-OLIVEIRA, Inês M. Climatologia: noções básicas e climas do Brasil. v. 1. São Paulo: Oficina de Texto, 2007.

PARK BJ, SIGEL K, VAZ V, KOMATSU K, MCRILL C, PHELAN M, et al. 2005. Uma epidemia de coccidioidomicose no Arizona associada às mudanças climáticas, 1998-2001. J InfectDis.

SHEPPARD, P.R.; COMRIE, A.C.; PACKIN, G.D.; ANGERSBACH, K.; HUGHES, M.K. O clima do sudoeste dos EUA. Clim Res. 2002; 21: 219-238.

SIH. Sistema de Informação Hospitalar. 2017. Consultas. Disponível em: <http://www2.datasus.gov.br/SIHD/consultas> Acesso em: 01 nov. 2017.

SILVA, F.B. et al. Evidências de Mudanças Climáticas na Região de Transição Amazônia-Cerrado no Estado do Maranhão. Revista Brasileira de Meteorologia, v. 31, n. 3, 330-336, 2016.

SOARES, V.Y; LUCIO FILHO, C.E; CARVALHO, L.I; SILVA, A.M; EULÁLIO, K.D. Análise clínica e epidemiológica de pacientes portadores de HIV / AIDS admitidos em um hospital de referência da Região Nordeste do Brasil .Rev Inst Med Trop São Paulo 2008 ; 50 : 327 - 32.

SPEARMAN, C.E. "Inteligência geral" objetivamente determinada e medida. American Journal of Psychology, v. 15, n. 2, $201-292,1904$.

ZHANG, W.Y. et al. Measuring mixing patterns in complex networks by Spearman rank correlation coeficiente. Physica A: Statistical Mechanics and its Applications. v. 451, 440-450, 2016. 